\section{Applied system innovation}

Mechanical engineering and design innovations are both academic and practical engineering fields that involve systematic technological materialization through scientific principles and engineering designs. Technological innovation by mechanical engineering includes IT-based intelligent mechanical systems, mechanics, and design innovations. IT-based intelligent mechanical systems, which implants intelligence to machine systems, is an interdisciplinary area combining conventional mechanical technology and new information technology. Since Internet of Things (IOT) has been widely studied in recent years, the topics relevant to applied system innovations for IOT are particularly interesting and may attract many excellent researchers' continued interest in the subject. Thus, a Special Collection in Advances in Mechanical Engineering titled "Applied System Innovation" was proposed this year.

In addition, the 2016 IEEE International Conference on Applied System Innovation (IEEE ICASI 2016) was held in Okinawa, Japan, on May 27 to 31, 2016. There were 520 papers from 10 countries presented in the conference. It provided a unified communication platform for researchers in a wide area of topics. This Special Collection selected 34 excellent papers on the topic of Applied System Innovation from IEEE ICASI 2016 and other high-quality papers that fit the aims and scope of this collection. The subjects of 34 papers include the following:

- Intelligent mechanical manufacturing systems;

- Mathematical problems on mechanical system design;
2018, Vol. 10(10)

(C) The Author(s) 2018

DOI: $10.1|77 /| 6878 \mid 4018805610$

journals.sagepub.com/home/ade

(SSAGE
- Smart electromechanical system analysis and design;

- Computer-aided methods for mechanical design procedure and manufacture;

- Computer and human-machine interaction;

- Internet technology on mechanical system innovation;

- Various computational topics of methodologies and procedures of design;

- Machine diagnostics and reliability;

- Human-machine interaction/virtual reality and entertainment.

\author{
Teen-Hang Meen ${ }^{1}$, Shoou-Jinn Chang ${ }^{2}$ and \\ Stephen D Prior ${ }^{3}$ \\ ${ }^{1}$ Department of Electronic Engineering, National \\ Formosa University, Huwei, Taiwan \\ ${ }^{2}$ Department of Electrical Engineering, National \\ Cheng Kung University, Tainan, Taiwan \\ ${ }^{3}$ Astronautics and Computational Engineering, \\ University of Southampton, Southampton, UK
}

\section{Acknowledgements}

The guest editors would like to thank the authors for their contributions to this Special Collection and all the reviewers for their constructive reviews. We are also grateful to Katrina Newitt, Senior Peer Review Manager at SAGE Publishing, for her time and efforts in the publication of this Special Collection for Advances in Mechanical Engineering. 